\title{
Fine-scale precipitation structure of a cold front and the problem of the representativeness error
}

\author{
S. Ivanov and J. Palamarchuk \\ Odessa State Environmental University, Ukraine \\ Received: 9 July 2006 - Revised: 6 January 2007 - Accepted: 6 January 2007 - Published: 26 April 2007
}

\begin{abstract}
The proper knowledge of spatial precipitation structure is important as much as that of microphysics processes for reliable quantitative precipitation forecasts. This study considers some aspects of how precipitation is organized on meso- and fine-scales, within a cold front line and how moisture transport is driven by these structures. Also, a spectral space vision of the representativeness error is proposed, which highlights uncertainties arising on the scales lying between resolutions of different networks. This approach is used to explain an improper simulation of humidity and precipitation fields in models whose resolution is coarser than the scales being considered.
\end{abstract}

\section{Introduction}

Precipitation is one of the most complicated physical processes in the atmosphere. It shows high variability in space and time. This fact imposes tight requirements on an observation network to be properly arranged. The existing rain gauge networks in most areas are rather coarse and not able to provide a detailed description of the precipitation structures especially on meso-scale. The weather radar network, on the other hand, operates by reflectivity with high temporal and spatial resolutions (Collier, 1986; Crum et al., 1998), but attempts to relate the measured reflectivity of the radar sampling volume to the surface rain rate can lead to substantial error by a factor of 2 (Austin, 1987). Additionally, strong nonlinear dynamics of the water cycle in the atmosphere are simplified and parameterized in numerical models, thus misrepresenting fields on a finite grid resolution. The above reasons result in an inadequate description of precipitation, in general, as well as improper forecasting of it. Furthermore, in contrast to other atmospheric variables, precipitation fields

Correspondence to: $\mathrm{S}$. Ivanov

(svvivo@ te.net.ua) are discontinuous, and their typical spatial scales are significantly smaller than, for example, those of temperature or geopotential fields. This also sufficiently limits techniques used for analysis of precipitation and does not allow us to properly describe processes associated with those unresolved scales.

Thus, the main focus of this study is to identify fine-scale precipitation structures that occur within a cold front and describe moisture and energy transport driven by them. This task is related to improving numerical prediction systems through better understanding of the precipitation physics, moisture and energy fluxes on synoptic and meso-scales.

\section{Spatial precipitation structure}

\subsection{Precipitation scales on a cold front}

Analyses of previous radar network observations have revealed that precipitation within atmospheric fronts show banded patterns (Browning, 1974; Kreitzberg and Brown, 1970; Houze and Hobbs, 1982; Hobbs et al., 1980): a frontal zone includes a few $30-70 \mathrm{~km}$ bands with intensive precipitation alternating with wider bands of $60-120 \mathrm{~km}$ with less intensive precipitation. The length of these bands is about $100 \mathrm{~km}$. NWP models currently with a horizontal resolution of the order of $10 \mathrm{~km}$, which resolves these large scale frontal features of precipitation (Jones and Macpherson, 1997). However, the complicate nature of precipitation on meso-scales and uncertainties in its spatial distribution on model grids lead to substantial misrepresentation of the fine scale precipitation and associated processes (such as moisture and energy fluxes) in simulations.

The present case study focuses on the cold front, which crossed Central Europe on 1-2 January 2005 (Fig. 1a). The weather in this region was determined by extensive deep low near Iceland and anticyclone rapidly moving from the Azores

Published by Copernicus GmbH on behalf of the European Geosciences Union. 


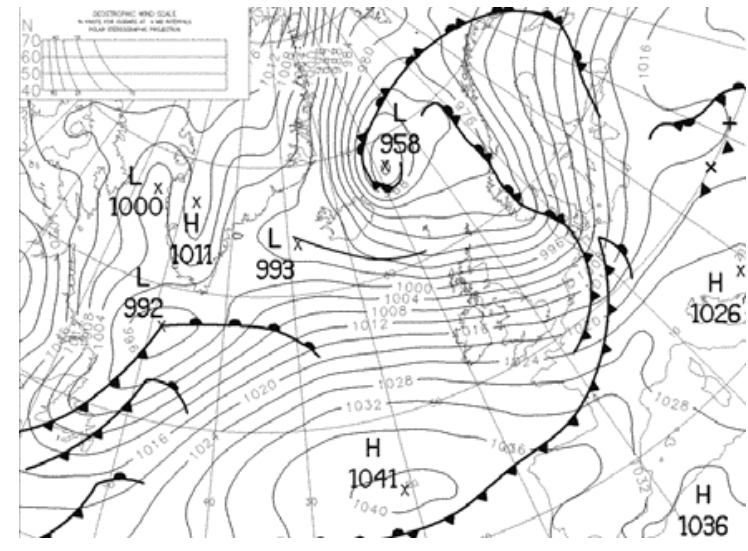

(a)

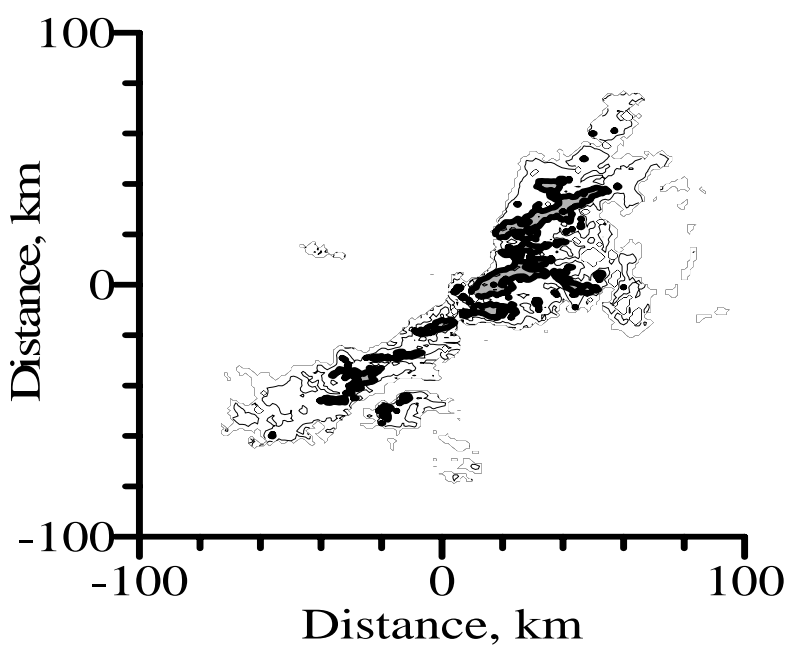

(b)

Fig. 1. (a) Analysis chart from UK MetOffice operational forecasts for 02/01/2005 00:00, (b) frontal precipitation from MIUB radar for $02 / 01 / 0500: 41$; the precipitation structures of radar reflectivity above $35 \mathrm{dBz}$ (approximately $5 \mathrm{~mm} / \mathrm{h}$ of precipitation rate) are shaded.

to South Europe. The thermodynamical conditions and upper level flow were favourable to sharpening of the polar front in the trough over the Northern Atlantic. The front showed a temperature contrast of 5-8 degrees and was moving with the speed of about $15 \mathrm{~m} / \mathrm{s}$. Deep cloudiness and strong rain showers accompanied its passage.

For the purpose of identification of meso- and fine-scale features on the front, high-resolution $(125 \mathrm{~m}$ radial and 1 degree azimuthal) X-band radar data are used (http://www. meteo.uni-bonn.de/forschung/gruppen/radar/). The Plan Position Indicator with a 5-min scan interval monitors an area of $100 \mathrm{~km}$ radius.

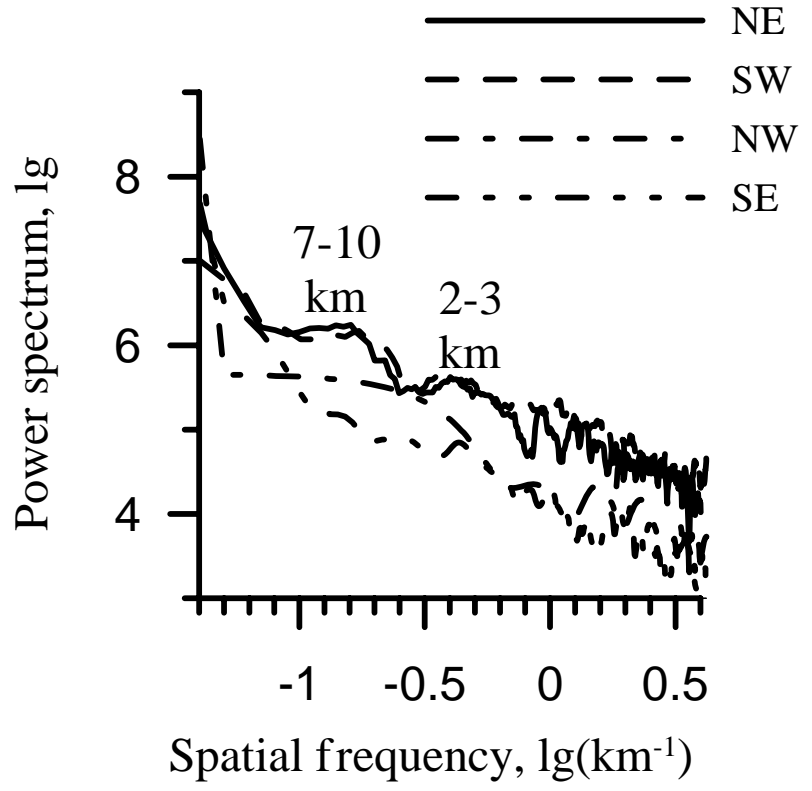

Fig. 2. The averaged spatial spectra of radar intensity at various directions. The north-east (NE) and south-west (SW) directions are in parallel with the front line, while the north-west (NW) and southeast (SE) directions are normal to the front line.

Spectral analysis has been applied to radar data in order to estimate dominant spatial variations of reflectivity and their characteristics in the frontal area, particularly parallel and normal to the front. Fortunately, the front passed over the exact radar location. It sufficiently simplified the use of the Fourier transforms to radar data collected along the beam lines. Such an approach is based on evenly distributed alongbeam data and provides results which are free from smearing, due to polar-Cartesian transform. Also, this method, in contrast to the statistical modelling of cloud and precipitation fields (Venema et al., 2006; Scheirer and Schmidt, 2005), allows us to consider asymmetric and heterotropic precipitation structures, for example, separately in parallel and normal to the front.

The main features of the precipitation structures are shown in Fig. $1 \mathrm{~b}$ and their spectral distribution along the front line are summarized in Fig. 2. All spectra display some "red noise" (Fraedrich and Ziehmann-Schlumbohm, 1994; Tanaka and Kimura, 1998), i.e. the longer the scale the larger the amplitude. The spectra can be separated in two groups. The first group includes the spectra computed along the front moving direction, i.e. northwestward and southeastwards. These spectra show that there are no dominant scales in these directions, and the power is about one order less than for the whole dataset. In fact, there are no significant regular precipitation structures ahead and behind the front line, but only the smallest (of size of $1 \mathrm{~km}$ ) and weakest solitary forms. The second group of spectra consists of the estimates of spatial 
variability along the front line that is in northeastward and southwestward directions. Unlike the first group, these spectra show two significant peaks exceeding the $95 \%$ confidence level. These peaks correspond to the front line formations, whose spatial dimensions are $2-3$ and $7-10 \mathrm{~km}$. The properties of spatial precipitation spectra have a power-law dependence on scale, in accordance with the multifractal paradigm (Turcotte, 1997) and its applications to the cloud sciences (Evans and Wiscombe, 2004; Scheirer and Schmidt, 2005), but also assume the existence of a few dominant scales (Mandelbrot, 1982).

Further, the larger structures are observed on the entire front line, while the smaller ones are mostly closer to the cyclone center and to the lesser extent to its periphery. Both groups of spectra also reflect the presence of smaller scale precipitation structures of order of $1 \mathrm{~km}$ and less, but their significance was not confirmed. The subsequent analysis, which is not considered here, showed that the temporal behavior of these smallest patterns is rather intermittent and inconsistent. One possible explanation of it, is that their lifetime is volatile and less than the scanning interval, i.e. $5 \mathrm{~min}$.

The above results provide robust estimates for the whole range of available spatial scales down to theoretical $250 \mathrm{~m}$. But they are obtained only for those scans when the front was located over the radar position. One may ask a question: how permanent are these precipitation structures during a front lifetime? To respond to this question, a selected domain with the dimension size of $130 \times 20 \mathrm{~km}$ moving together with the frontal precipitation patterns is also considered, in order to provide a better understanding of evolutions of the rain structures.

This procedure includes the coordinate transform and smoothing of the original precipitation field, from radar data for the homogeneous coverage on a regular net. The smoothing radius is chosen so that it ensures that the polar-toCartesian transform does not result in the loss of any grid points. Thus, to meet this requirement at the outer area of radar measurement, the radius should be at least about 800 $900 \mathrm{~m}$. This value suppresses the smallest and intermittent precipitation structures but does not sufficiently affect larger scales. The spectra estimated in such a way are similar to those shown in Fig. 2 for the northeast and southwest directions, i.e., they indicate the presence of the significant spatial structures of 2-3 and 7-10 km scales, but cut-off smaller structures smoothed by the coordinate transform procedure.

Further, phase relations demonstrate that these wave-like structures move along the front line and are responsible for the moisture transfer at that direction. This will be discussed in the next section.

\subsection{Moisture transport}

Another result outlined in this study is that the above finescale structures are responsible for the moisture transport along the front line. The use of the threshold value of $35 \mathrm{dBZ}$

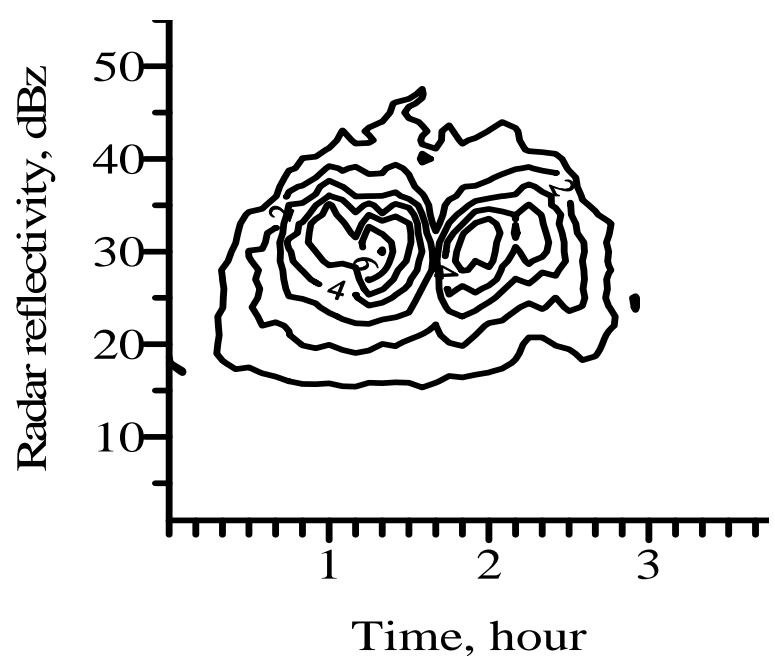

(a)

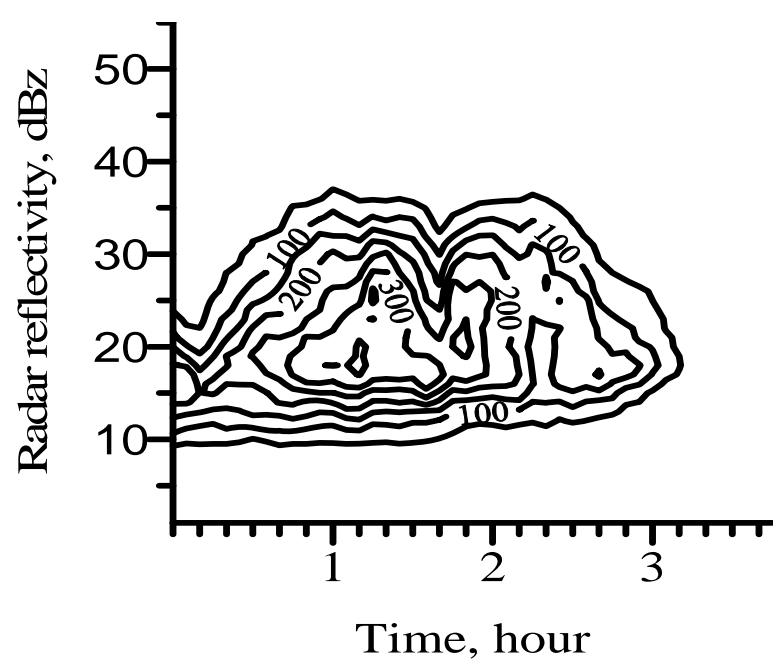

(b)

Fig. 3. (a) The water contents (in $\times 10^{5} \mathrm{~m}^{3}$ ) (a) and (b) area (in $\mathrm{km}^{2}$ ) associated with different radar reflectivity (precipitation rate) during the cold front event.

for reflectivity allowed us to pinpoint the trajectories of precipitation maxima. These trajectories deviated anticlockwise from the front motion by an angle of 40-50 degrees. Such behavior can be considered as the relative motion of the precipitation structures along the moving front. This process accounts for moisture transport from the periphery of a cyclone towards its center, with subsequent release of energy in the form of latent heat fluxes. This process may reinforce a cyclonic circulation throughout the upscale energy exchange. 


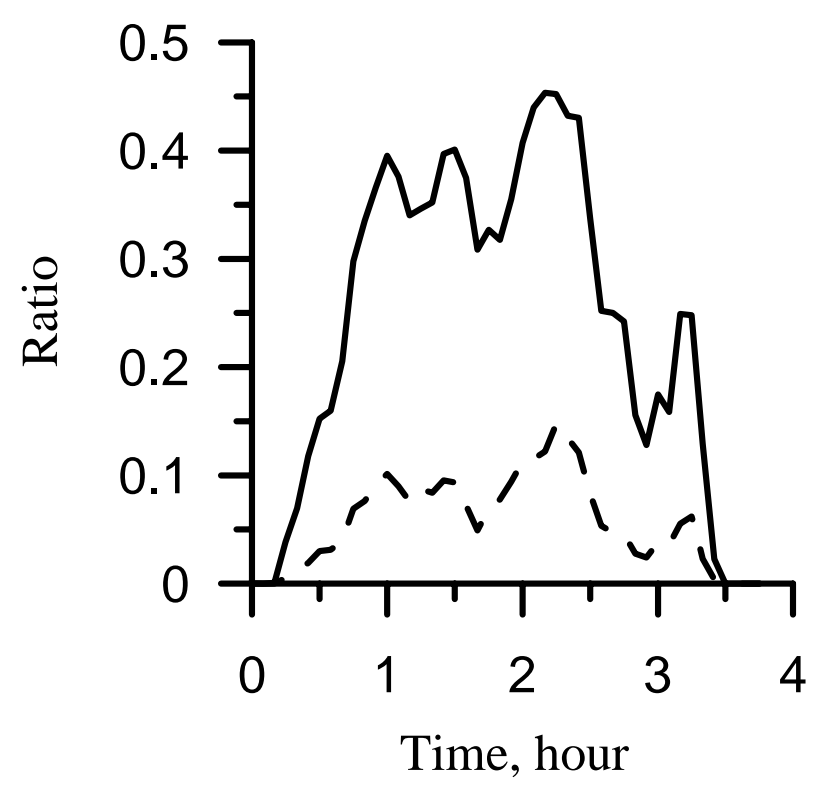

Fig. 4. The ratio of water contents (solid) and area (dash) included into the structures with the precipitation rate above by $5 \mathrm{~mm} / \mathrm{h}$ to total corresponding values of the cold front during the event.

The following example illustrates the importance of the meso-scale precipitation structures for the moisture transport. Figure 3 shows the temporal change of the water content and the area covered by precipitation of different rate versus radar reflectivity. The maximum of water content within the front is associated with cells of relatively high rain rate values of over $5 \mathrm{~mm} / \mathrm{h}$ (approximately $35 \mathrm{dBz}$ of radar reflectivity). They contain about $50 \%$ of total frontal rain water (Fig. 4) but occupy only $10-15 \%$ of the frontal domain, while the maximum of the spatial coverage corresponds to less intensive precipitation with rates about $0.3-0.7 \mathrm{~mm} / \mathrm{h}$ (15-20 dBz) (Fig. 3b). Thus, mass and energy fluxes associated with those meso-scale precipitation structures are threefour times higher than the averaged background estimates for the front. This well known fact, however, is still not properly simulated in models (Claussen, 1991; Roach, 1997; Gilmore et al., 2004) because of two reasons. First, models fail to reproduce strong non-linearity in severe storms. Second, the so-called effective values (Roach, 1998), i.e., the averaged values for the model grid, are sensitive to resolution.

These results are rather descriptive and definitely require a comprehensive diagnosis and quantitative estimates of fluxes on the above scales, which will be a focus of further research. Nevertheless, at the moment, they highlight the necessity to properly account for the processes on sub-grid scales, especially when their energetic characteristics are several times higher than the background. These details are not included in simplified parameterization schemes for a coarse resolution run (see, for example, Zehnder, 2001). The parameterization schemes for microphysics (Tao and Simpson, 1993;
Schultz, 1995; Reisner et al., 1998) used in current operational forecasting models simulate meso-scale precipitation structures of extra-tropical fronts, but the spatial distribution and magnitude of these structures are still improper. On the other hand, the importance of this problem and the potential misrepresentation caused by data assimilated from networks of different resolution, are explained in the next section.

\section{The representativeness error problem}

In this section, we attempt to estimate a potential contribution of the network's resolution as one source of error. As pointed out by Palmer (2001) and Jung (2005), the methodology used to approximate the equations of motion by neglecting the variability of unresolved scales is itself a source of systematic errors. This is a difficult problem because the error is never observed directly and can only be estimated in a statistical sense. Moreover, decomposition of the error into its components depending on the sources seems to be a very theoretical task, based on certain conjectures.

The representativeness error problem arises when precipitation estimates from different data sources at different resolutions are compared, i.e., from satellites with a resolution of $20-30 \mathrm{~km}$ and radars with a resolution of about $100 \mathrm{~m}$ to a raingauge network with a separation between stations of about $150 \mathrm{~km}$ and often to a model grid resolution from a few $\mathrm{km}$ to tens and hundreds $\mathrm{km}$. Thus, the multi-scale inherent characteristics of atmospheric flow become to be scaledependent on the resolution of a data source. Mahfouf and Noilhan (1991) proposed to avoid the representativeness error of the on-site estimates by deploying several instruments within a relatively small area. This approach increases the cost of the measurements but only narrows the limits toward the size of the area. Tustison et al. (2001) pointed out the importance of taking into account the multi-scale variability of precipitation. They showed that the resolution of data introduces the representativeness error, which is non-zero even in cases of perfect model or instrumental error estimates. Bormann et al. (2003) estimate the parts of the observation error by extrapolating the separation/correlation relationship to zero separation on the base of the observational method (Hollingsworth and Lonnberg, 1986).

We propose an approach in which the precipitation observation error $\varepsilon$ includes the following components. First, the instrumental error $\varepsilon^{\text {ran }}$ takes into account known or suggested statistics. For direct observations these statistics satisfy Gaussian distribution (mean zero and unity variance) (Hollingsworth and Lonnberg, 1986), while remotely sensed data, i.e. those obtained from radars and satellites, may contain certain bias (Eyre, 1997). Nevertheless, the instrumental error is a priori determined and stable, and relates to the instrumental quality.

The second part is the representativeness error $\varepsilon^{\text {rep }}$. It means a measure of accuracy with which the data gathered 
at a single point are able to describe a field over some area around the point. This error comes from the fact that the spatial separations between observation points exceed the spatial variability of the atmospheric properties; hence the latter is not captured by the observational network. The impact from unresolved atmospheric variability is manifested in spurious oscillations superimposed on the data in form of some noise. These oscillations do not correspond to any real physical process and introduce different errors to data obtained from different resolution networks, even if the same instruments are used in those networks. This happens because the unresolved atmospheric scales are of continuous nature while we intend to reproduce them by means of discretization whose step is larger than those scales. In the spectral analysis this problem is well-known as the Gibbs effect (Chen and Kuo, 1992), when high-frequency unresolved components manifest themselves in the form of false components, which are accounted for by numerical effects rather than natural phenomena. The error statistics of those components are far from to be known. We might assume that the statistics include some residual spatial correlations. One would like to assess how strongly this representativeness error is related to the observation net resolution. The expectation of total observation error covariance stratified against separation at locations $i$ and $j$ has the following form

$$
\begin{aligned}
\overline{\varepsilon_{i} \varepsilon_{j}} & =\overline{\left(\varepsilon_{i}^{\mathrm{ran}}+\varepsilon_{i}^{\mathrm{rep}}\right)\left(\varepsilon_{j}^{\mathrm{ran}}+\varepsilon_{j}^{\mathrm{rep}}\right)}= \\
& =\overline{\varepsilon_{i}^{\mathrm{ran}} \varepsilon_{j}^{\mathrm{ran}}}+\overline{\varepsilon_{i}^{\mathrm{ran}} \varepsilon_{j}^{\mathrm{rep}}}+\overline{\varepsilon_{i}^{\mathrm{rep}} \varepsilon_{j}^{\mathrm{ran}}}+\overline{\varepsilon_{i}^{\mathrm{rep}} \varepsilon_{j}^{\mathrm{rep}}}= \\
& =\overline{\varepsilon_{i}^{\mathrm{ran}} \varepsilon_{j}^{\mathrm{ran}}}+0+0+\overline{\varepsilon_{i}^{\mathrm{rep}} \varepsilon_{j}^{\mathrm{rep}}}
\end{aligned}
$$

If one assumes that there is no correlation between the random instrumental error at one location and the representativeness error at the other location, then the two middle terms on the second line vanish.

If $i=j$, i.e. we consider the total observation error covariance at overlaying locations, then one can expect that the representativeness error diminishes, as the smallest scales are resolved. The error covariance matrix then becomes

$\overline{\varepsilon_{i} \varepsilon_{j}}=\overline{\varepsilon_{i} \varepsilon_{i}}=\overline{\varepsilon_{i}^{\mathrm{ran}} \varepsilon_{i}^{\mathrm{ran}}}=\sigma_{i}^{2}$

i.e., we obtain the pure statistics for the instrumental error.

If $i \neq j$, i.e. we take into account the potential contribution of unresolved scales to the total error, then the error covariance is also defined by the representativeness part of the error

$\overline{\varepsilon_{i} \varepsilon_{j}}=\sigma_{i}^{2}+\left(\overline{\varepsilon_{i}^{\mathrm{rep}}}\right)^{2}$

Figure 5 shows the spectral space vision of this problem implemented for the case when data from two and more informational sources of different resolutions are blend in one batch. This is the case of data assimilation. It follows that the contribution of the representativeness error toward the larger

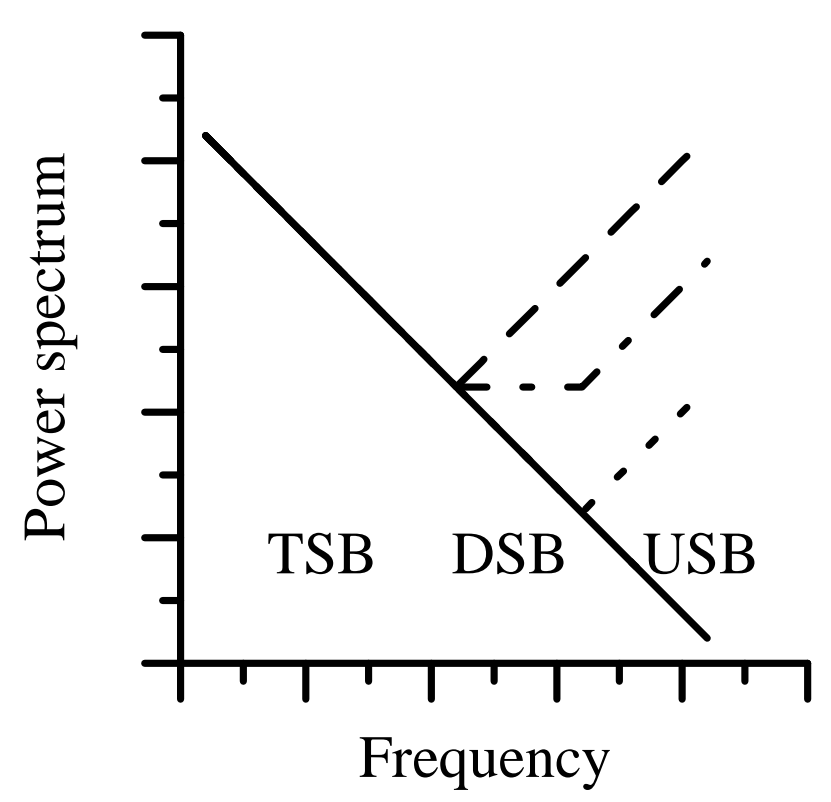

Fig. 5. Spectral space vision of the representativeness error problem. The solid line is a "true" spectrum, dash line is a coarseresolution estimate of "true" spectrum, dot line is a fine-resolution estimate, dash-dot line is resulted estimate. TSB means the "true scale band", DSB is the "disturbed scale band", USB is the "unresolved scale band".

separations increases, when a background cascade distribution of energy on a range of scales is implied. Additionally, it shows that the band of scales between the coarse and fine resolutions is subject to major distortions. This example is very illustrative because it shows that the quality of numerical forecast might depend on the choice of a model grid in addition to physical and dynamical model formulations. The knowledge of scales of predominant precipitation mesostructures would help us to make a proper choice of model resolution and reduce the forecast error.

\section{Conclusions}

The characteristics and behaviour of meso-scale precipitation structures on a cold front have been studied in this research. Two organized wave-like patterns of rain intensity with scales of 2-3 and 7-10 km were identified. The mesoscale precipitation structures with the maximum rain rate contain about half of the moisture brought with the front, while their total size does not exceed $10-15 \%$ of the frontal precipitation area. These patterns move along the front line from the cyclone periphery toward its centre. As a result, the trajectories of precipitation maxima deviate from the front direction by about 40-50 degrees anticlockwise. We contend that such meso-scale precipitation is important for accounting for mass and energy balances and should be properly 
described in model formulations. The forecast error may increase due to the increase in the representativeness component, if the model resolution is incompatible with unresolved processes. The source of the representativeness error is also described.

Edited by: S. C. Michaelides and E. Amitai

Reviewed by: anonymous referees

\section{References}

Austin, P. M.: Relation between measured radar reflectivity and surface rainfall, Mon. Wea. Rev., 115, 1053-1070, 1987.

Bormann, N., Saarinen, S., Kelly, G., and Thépaut, J.-N.: The spatial structure of observation errors in atmospheric motion vectors from geostationary satellite data, Mon. Wea. Rev., 131, 706-718, 2003.

Browning, K. A.: Mesoscale structure of rain system in the British Isles, J. Meteorol. Soc. Japan, 11, 52, 314-327, 1974.

Claussen, M.: Estimation of areally averaged surface fluxes, Boundary-Layer Meteorol., 54, 387-410, 1991.

Chen, Q. and Kuo, Y.: A harmonic-sine series expansion and its application to partitioning and reconstruction problems in a limited area, Mon. Wea. Rev., 120, 91-112, 1992.

Collier, C. G.: Accuracy of radar estimates by radar. Part I: Calibration by telemetring raingauges, J. Hydrol., 83, 207-223, 1986.

Crum, T. D., Saffle, R. E., and Wilson, J. W.: An update on the NEXRAD program and future WSR-88D support to operations, Wea. Forecast., 13, 253-262, 1998.

Evans, K. F. and Wiscombe, W. J.: An algorithm for generating stochastic cloud fields from radar profile statistics, Atmos. Res., 72, 263-289, 2004.

Eyre, J. P.: Variational assimilation of remotely-sensed observations of the atmosphere, J. Meteorol. Soc. Japan, 75, 331-338, 1997.

Fraedrich, K. and Ziehmann-Schlumbohm, C.: Predictability experiments with persistence forecasts in a red-noise atmosphere, Q. J. R. Meteorol. Soc., 120, 387-428, 1994.

Gilmore, M. S., Straka, J. M., and Rasmussen, E. N.: Precipitation uncertainty due to variations in precipitation particle parameters within a simple microphysics scheme, Mon. Wea. Rev., 132, 2610-2627, 2004.

Hobbs, P. V., Matejka, T. J., Herzegh, P. H., Locatelly, J. D., and Houze, R. A.: The meso-scale and microscale structure and organization of clouds and precipitation in midlatitude cyclones. Part I. A case study of a cold front, J. Atmos. Sci., 37, 568-596, 1980.

Hollingsworth, A. and Lonnberg, P.: The statistical structure of short-range forecast errors as determined from radiosonde data, Tellus, 38A, 111-136, 1986.

Houze, R. A. and Hobbs, P. V.: Organization and structure of precipitating cloud systems, Adv. Geophys., 24, 225-315, 1982.
Jones, C. D. and Macpherson, B.: A latent heat nudging scheme for the assimilation of precipitation data into an operational Mesoscale model, Meteorol. Appl,, 4, 269-277, 1997.

Jung, T.: Systematic errors of the atmospheric circulation in the ECMWF forecasting system, Q. J. R. Meteorol. Soc., 131, 10451073, 2005.

Kreitzberg, C. W. and Brown H. A.: Mesoscale weather systems within an occlusion, J. Appl. Meteorol., 9, 417-432, 1970.

Mahfouf, J.-F. and Noilhan, J.: Comparative study of various formulations of evaporation from bare soil using in-situ data, J. Appl. Meteorol., 30, 1354-1365, 1991.

Mandelbrot, B. B.: The Fractal Geometry of Nature, W H Freeman, New York, USA, 460 p., 1982.

Palmer, T. N.: A nonlinear dynamical perspective on model error: A proposal for non-local stochastic-dynamic parameterization in weather and climate prediction models, Q. J. R. Meteorol. Soc., 127, 279-304, 2001.

Reisner, J., Rasmussen R. J., and Bruintjes R. T.: Explicit forecasting of supercooled liquid water in winter stroms using the MM5 mesoscale model, Quart. J. Roy. Meteor. Soc., 124B, 1071-1107, 1998.

Roache, P. J.: Quantification of Uncertainty in Computational Fluid Dynamics, J. Fluid Mech., 29, 123-160, 1997.

Roache, P. J.: Verification and Validation in Computational Science and Engineering, Hermosa Publishers, Albuquerque, New Mexico, 1998.

Scheirer, R. and Schmidt, S.: CLABAUTAIR: a new algorithm for retrieving three dimensional cloud structure from airborne microphysical measurements, Atmos. Chem. Phys., 5, 2333-2340, 2005 , http://www.atmos-chem-phys.net/5/2333/2005/.

Schultz, P.: An explicit cloud physics parameterization for operational numerical weather prediction, Mon. Wea. Rev., 123, 33313343, 1995.

Tao, W.-K. and Simpson, J.: Goddard Cumulus Ensemble Model. Part I: Model Description. Terrestrial, Atmos. Oceanic Sci., 4, 35-72, 1993.

Tanaka, H. L. and Kimura, K.: Time series analysis of natural variability in barotropic energy of the atmosphere with ECMWF global analysis, J. Meteorol. Soc. Japan, 76, 267-274, 1998.

Turcotte, D. L.: Fractals and Chaos in Geology and Geophysics, Cambridge University Press, U.K., 412p., 1997.

Tustison, B., Harris, D., and Foufoula-Georgiou, E.: Scale issues in verification of precipitation forecasts, J. Geophys. Res., 106, 11775-11 784, 2001.

Venema, V., Meyer, S., Gimeno García, S., Kniffka, A., Simmer, C., Crewell, S., Löhnert, U., Trautmann, U., and Macke, A.: Surrogate cloud fields generated with the Iterative Amplitude Adapted Fourier Transform algorithm, Tellus 58A, 104-120, 2006.

Zehnder, J. A.: A comparison of convergence- and surface-fluxbased convective parameterizations with applications to tropical cyclogenesis, J. Atmos. Sci., 58, 283-301, 2001. 